\title{
Accuracy of Ultrasound Estimated Fetal Weight in Small for Gestational Age and Appropriate for Gestational Age Grown Periviable Neonates
}

\author{
Adriane Haragan, $\mathrm{MD}^{1} \quad$ Katherine Himes, MD, MS ${ }^{1}$ \\ ${ }^{1}$ Division of Maternal-Fetal Medicine, Department of Obstetrics and \\ Gynecology, Magee-Womens Hospital, Pittsburgh, Pennsylvania \\ Am J Perinatol 2018;35:703-706. \\ Address for correspondence Adriane Haragan, MD, Division of \\ Maternal-Fetal Medicine, Department of Obstetrics and Gynecology, \\ Magee-Womens Hospital, 300 Halket Street, Pittsburgh, PA 15213 \\ (e-mail: haraganaf@upmc.edu).
}

\author{
Abstract \\ Keywords \\ - periviable birth \\ - preterm birth \\ - estimated fetal \\ weight \\ - small for gestational \\ age \\ - ultrasound \\ - accuracy of \\ ultrasound \\ - birth weight
}

Objective Clinicians use estimated fetal weight (EFW) as a proxy for birth weight (BW) in the antenatal period. Our objective was to compare the accuracy of EFW obtained by ultrasound to BW among infants born during the periviable period and determine if accuracy of EFW varied among small for gestational age (SGA) versus appropriate for gestational age (AGA) grown neonates.

Study Design We included women who delivered between $23^{0 / 7}$ and $25^{6 / 7}$ weeks' gestation and had an EFW within 7 days of delivery. Mean percentage difference and median absolute percentage difference between EFW and BW were calculated.

Results Our cohort included 226 neonates with a mean gestational age of $24^{1 / 7} \pm 0.8$ weeks and median BW of $653 \mathrm{~g}$ (interquartile range [IQR]: $580-750 \mathrm{~g}$ ). The median absolute percentage difference between EFW and BW of fetal weight estimates was 9.2\% (IQR: 3.6-17.2). EFW overestimated BW for $75 \%(n=171)$ of the cohort. Among SGA infants, the mean percentage difference in EFW and BW was $16.2 \pm 19.4 \%$ versus $6.9 \% \pm 13.1 \%$ in AGA infants $(p=0.019)$.

Conclusion EFW overestimated BW in this cohort. In addition, ultrasound was less accurate among infants born SGA. These data are important to consider when counseling families facing periviable delivery.
Consensus statements from the American Academy of Pediatrics and the Society for Maternal-Fetal Medicine have emphasized the importance of providing families facing periviable birth with the most accurate prognostic data available regarding infant and child outcomes. ${ }^{1,2}$ Birth weight (BW) is an important predictor of outcomes among infants born during the periviable period. The National Institute of Child Health and Human Development includes BW as one of the variables in its algorithm to calculate outcome predictions for extremely preterm infants. ${ }^{3}$ While this is a widely used tool to augment antenatal counseling, obstetricians and neonatologist must

received

March 11, 2017

accepted after revision

November 29, 2017

published online

December 29, 2017 use ultrasound-derived estimated fetal weight (EFW) as a proxy for BW.

Data are limited regarding the accuracy of ultrasoundderived EFW to predict BW in extremely preterm neonates. The American Congress of Obstetricians and Gynecologists states that published formulas for estimating fetal weight show good correlation to BW; however, variability of estimates is up to $20 \%$ within the various equations. ${ }^{4-7}$ The accuracy of fetal weight estimates has been studied extensively for several decades and the literature suggests that the absolute error rate of ultrasound ranges between 2 and $12 \%$
Copyright $\odot 2018$ by Thieme Medical Publishers, Inc., 333 Seventh Avenue, New York, NY 10001, USA. Tel: +1(212) 584-4662.
DOI https://doi.org/ 10.1055/s-0037-1617433. ISSN $0735-1631$. 
in appropriate for gestational age (AGA) grown fetuses. ${ }^{6,7}$ Only two published studies have examined infants born during the periviable period. These demonstrated comparable accuracy with median absolute percentage errors ranging from 8.8 to $9.4 \%$. These studies, however, had small sample sizes, with 134 infants included in the largest study. ${ }^{8,9}$ Furthermore, there were discrepant findings regarding the relationship between the accuracy of EFW and BW in extremely preterm neonates. Of the two studies comparing EFW to BW in this population, one found no clinically significant relationship between the accuracy of EFW and BW, while the other found that the accuracy of EFW was significantly less among small for gestational age (SGA) infants. $^{8,9}$

The purpose of our investigation was to evaluate the accuracy of EFW obtained by ultrasound to predict BW by determining both the percentage difference between EFW and BW, and the absolute difference between EFW and BW among infants born during the periviable period. Furthermore, we sought to determine if percentage difference varied between SGA and AGA grown neonates and among infants with a BW less than $500 \mathrm{~g}$.

\section{Materials and Methods}

Our study population included all nonanomalous live-born infants born between $23^{0 / 7}$ and $25^{6 / 7}$ weeks' gestation at Magee-Womens Hospital in Pittsburgh, Pennsylvania from January 2005 to January 2014. Data on maternal, fetal, and neonatal outcomes were obtained from the Magee Obstetric Medical and Infant (MOMI) Database. Ultrasound EFW and pre-pregnancy body mass index (BMI) were abstracted directly from the medical record, as these variables are not included in the MOMI database. SGA was defined as a BW less than the 10th percentile based on Alexander BW curves. ${ }^{10}$ This study was approved by the University of Pittsburgh's Institutional Review Board.

Registered diagnostic medical sonographers performed all sonographic examinations and a faculty member of the Division of Maternal-Fetal Medicine at Magee-Womens Hospital reviewed all ultrasounds. The ultrasounds were performed in the outpatient diagnostic center using Phillips ATL HDI 4000 or Phillips HDI 5000 machines within 7 days of delivery. EFW was calculated using the Hadlock-3 formula $(\log 10$ weight $=1.3596-0.00386 \mathrm{AC} \times \mathrm{FL}+0.0064 \mathrm{HC}$ $+0.00061 \mathrm{BPD} \times \mathrm{AC}+0.0424 \mathrm{AC}+0.174 \mathrm{FL}$ ) incorporating the fetal head circumference, biparietal diameter, femur length, and abdominal circumference. ${ }^{4}$

The accuracy of fetal weight estimation was defined as the mean percentage difference between fetal weight estimates and BW using the formula $(\mathrm{EFW}-\mathrm{BW}) / \mathrm{BW} \times 100 \%$. With this formula, a negative mean percentage difference indicates that EFW on average underestimated the actual BW, whereas positive values indicate overestimation. We also calculated the median absolute percentage difference between EFW and BW using the formula: $|(E F W-B W) / B W \times 100|$. The absolute percentage difference gives a better sense of the central tendency of the error of fetal weight estimates as the distribu- tion does not cross zero given that this is an absolute value. Excess error in fetal weight estimates was defined as an absolute percentage difference between EFW and BW that was $\geq 20 \%$, as it was felt that smaller differences would be less likely to affect clinical decision making and this value has been used in comparative studies. $^{8}$

Descriptive statistics were used to characterize the cohort. The distribution of the accuracy of fetal weight estimates (difference in EFW and BW) and the absolute difference in EFW and BW were determined with skewness/kurtosis tests and $\mathrm{Q}-\mathrm{Q}$ plots. The absolute difference in fetal weight estimates and BW was not normally distributed and thus medians and interquartile ranges (IQRs) are presented and Kruskal-Wallis' test was used for analysis. The accuracy of fetal weight estimates was normally distributed without transformation. The association between the accuracy of EFW and BW was evaluated with univariate and multivariate linear regressions. Maternal pre-pregnancy BMI was included in the model given its strong a priori plausibility to affect the accuracy of ultrasound-derived fetal weight estimates. Interval from ultrasound to delivery was additionally included in the model given its significance in the univariate analysis. Categorical variables were compared with chi-squared. All statistical analyses were performed with STATA software (version 13.0).

\section{Results}

A total of 226 neonates delivered to 222 women during the study period. The clinical and demographic characteristics of the cohort are summarized in -Table 1. Approximately 20\% of the cohort was of advanced maternal age ( $\geq 35$ years) and $43 \%$ was obese (BMI $\geq 30 \mathrm{~kg} / \mathrm{m}^{2}$ ). The median interval from ultrasound to delivery was 2 days (IQR: 1-4 days). Eleven percent $(n=25)$ of neonates were classified as SGA and $10 \%$ $(n=23)$ had a BW less than 500 g. Preterm labor with progressive preterm delivery accounted for $74 \%$ of deliveries, while iatrogenic deliveries for preeclampsia with severe features accounted for the remainder of the preterm births.

The mean percentage difference of fetal weight estimates was $8.0 \pm 14.2 \%$ (-Fig. 1). The median absolute percentage

Table 1 Demographics

\begin{tabular}{|l|l|}
\hline Demographics & \\
\hline Maternal age $(\mathrm{y})$ & $27.7 \pm 6.3$ \\
\hline Mean gestational age at birth $(\mathrm{wk})$ & $24^{1 / 7} \pm 0.8$ \\
\hline Mean body mass index $\left(\mathrm{kg} / \mathrm{m}^{2}\right)$ & $30.5 \pm 7.4$ \\
\hline Mean estimated fetal weight $(\mathrm{g})$ & $713.3 \pm 136.9$ \\
\hline Mean birth weight $(\mathrm{g})$ & $667.7 \pm 135.7$ \\
\hline Nulliparous & $44 \%(n=98)$ \\
\hline Race & $30 \%(n=67)$ \\
\hline Black & $66 \%(n=147)$ \\
\hline White & $4 \%(n=8)$ \\
\hline Other &
\end{tabular}




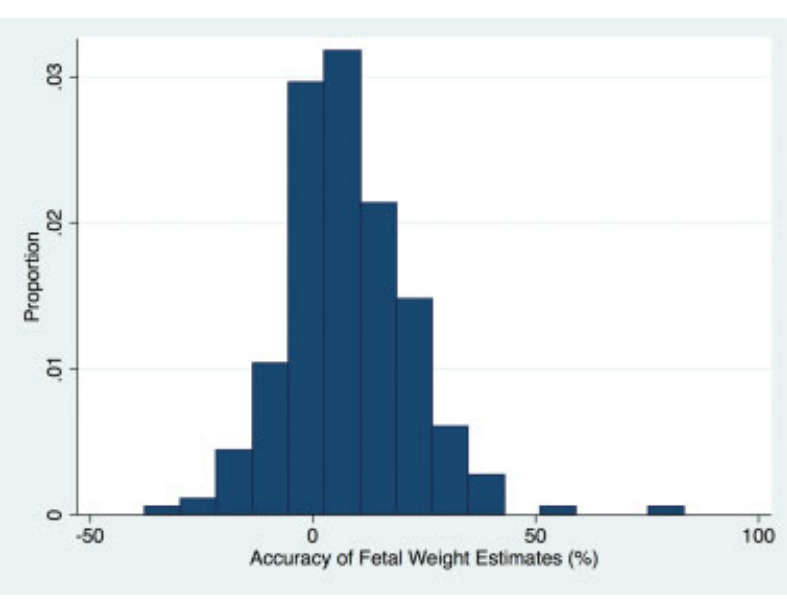

Fig. 1 Accuracy of fetal weight estimates to predict birth weight among infants born during the periviable period. Accuracy is defined as the mean percentage difference between fetal weight estimates and birth weight using the formula (EFW - birth weight)/birth weight $\times 100 \%$. With this formula, a negative mean percentage difference indicates that EFW on average underestimated the actual birth weight, whereas positive values indicate overestimation. EFW, estimated fetal weight.

difference between EFW and BW of fetal weight estimates was 9.2\% (IQR: 3.6-17.2). Importantly, ultrasound overestimated BW for $75.7 \%(n=171)$ of the cohort. In $19 \%$ of the cohort, ultrasound demonstrated excess error with an absolute percentage difference in fetal weight estimates compared with BW more than 20\%. Excess error was more likely in scans that over EFW. When fetal weight estimates overestimated BW, $22.2 \%$ of them had excess error $(\geq 20 \%)$ versus only $7.3 \%$ of ultrasounds where EFW underestimated BW $(p=0.013)$.

We next sought to examine the relationship between the accuracy of EFW and BW. There was a statistically significant inverse relationship between the accuracy of EFW and BW such that ultrasound estimates of fetal weight were less accurate as BW decreased (-Fig. 2). This remained significant when controlling for interval between ultrasound and

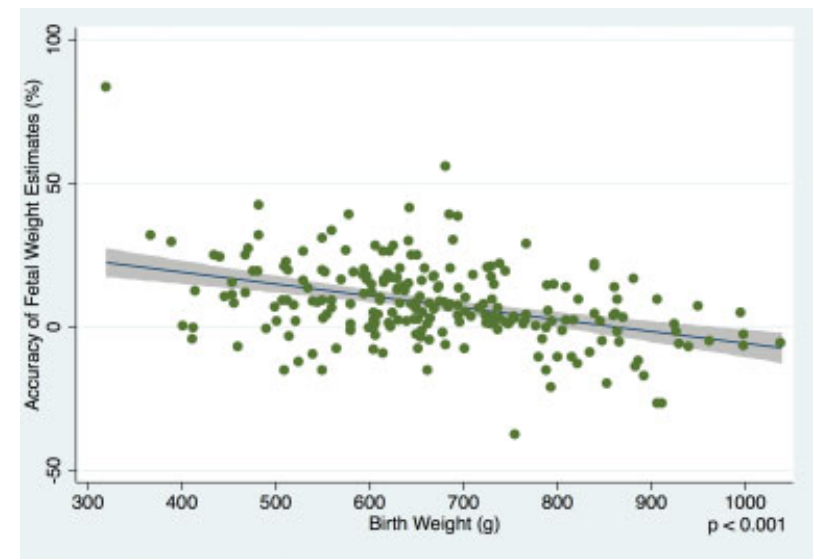

Fig. 2 Relationship between accuracy of fetal weight estimates and birth weight among infants born during the periviable period. The solid line represents the point estimates and the gray shaded area represents the $95 \%$ confidence interval. delivery as well as maternal pre-pregnancy BMI. Importantly, the systematic overestimation of fetal weight estimates is illustrated in -Fig. 2 as the majority of the values are above zero.

In our final analysis, we evaluated both the absolute difference in EFW and BW as well as the mean accuracy of fetal weight estimates among SGA infants and infants born less than $500 \mathrm{~g}$. The median absolute difference between fetal weight estimates and BW among SGA infants was more than AGA grown infants with a median absolute difference of 12.3\% (IQR: 7.0-24.8) compared with 8.6\% (IQR: 3.5-16.2). This result did not reach statistical significance $(p=0.062)$. Among infants born less than $500 \mathrm{~g}$, the median absolute percentage difference was significantly greater than those more than $500 \mathrm{~g}$ at delivery-15.2\% (IQR: 7.0-27.2) versus 8.6\% (IQR: $3.4-16.7 \%)(p=0.021)$. As discussed previously, the absolute value of the difference in EFW and BW does not depict the directionality of the error in ultrasound, and thus we explored the mean accuracy of fetal weight estimates among SGA infants and infants born less than $500 \mathrm{~g}$. Among SGA infants, the mean percentage difference in EFW and BW was $16.2 \pm 19.4 \%$ versus $6.9 \pm 13.1 \%$ in AGA infants $(p=0.019)$. Infants born less than $500 \mathrm{~g}$ also had a greater mean percentage difference in EFW and BW-18.2 $\pm 19.3 \%$ versus $6.7 \pm 13.0 \%(p=0.003)$.

Finally, we sought to determine if infants born SGA or less than $500 \mathrm{~g}$ were more likely to have excess error in fetal weight estimates than normally grown infants or those born more than 500 g. Approximately one-third (32\%) of infants born SGA had excess error in fetal weight estimates compared with $16.9 \%$ of AGA grown infants ( $p=0.067)$. Infants with a BW less than $500 \mathrm{~g}$ had excess error in fetal weight estimates in $39 \%$ of sonograms compared with $16.2 \%$ of infants who weighed more than $500 \mathrm{~g}(p=0.008)$.

\section{Comment}

The purpose of our investigation was to evaluate the accuracy of EFW to predict BW in periviable infants. We additionally hoped to determine if this difference varied among SGA neonates and those with a BW less than $500 \mathrm{~g}$, as this cohort has the highest risk for poor outcomes. An overestimation of BW in this group of neonates could mislead providers about their prognosis, thus providing families with inaccurate information. In fact, we found that ultrasound overestimated BW in more than $75 \%$ of our periviable neonates. The ultrasound-derived estimates of fetal weight were consistently less accurate as BW decreased. This was particularly true in SGA neonates, as almost one-third (32\%) had excessive ultrasound error in the prediction of their BW. This became statistically significant in neonates less than $500 \mathrm{~g}$.

Our study adds to the limited body of literature investigating the accuracy of EFW to predict BW in periviable neonates, particularly infants born SGA or at the extremes of viable weights. Our finding of an absolute percentage difference of $9.2 \%$ between EFW and BW is consistent with other studies investigating the accuracy of EFW to predict BW in low BW neonates and periviable neonates. ${ }^{8,9,11}$ To 
date, there have been two studies that have specifically investigated the use of EFW to predict BW in periviable neonates. ${ }^{8,9}$ Consistent with our results, both these studies showed that fetal weight estimates by ultrasound tend to overestimate BW. The relationship between the accuracy of fetal weight estimates and BW, however, has not been consistent in the extant literature. Ethridge et al found no relationship between the accuracy of EFW and BW, while Stefanelli and Groom found that the accuracy of EFW was less among SGA neonates. ${ }^{8,9}$ Our findings agree with the latter, as we also demonstrated that fetal weight estimates are less accurate among SGA neonates and infants born at the extremes of viable neonatal weights.

Our study has several strengths. The sample size of our cohort is larger than any other study to date with close to twice the number of infants as prior investigations. In addition, our cohort is a contemporary population with ultrasound images obtained by highly trained individuals with standardized protocols for image acquisition and a short ultrasound-to-delivery interval. Our study, however, also has important limitations. Though our cohort is the largest to date, the sample size remains relatively smallparticularly within the SGA cohort-perhaps limiting the ability to extrapolate our data to different populations, and accounts for the wide confidence intervals within this analysis. In addition, our sample is heterogenous with the inclusion of two twin gestations. However, we felt that including these neonates in the analysis is acceptable, as recent studies have shown that twin growth does not meaningfully differ from singleton growth until 32 weeks' gestation. ${ }^{12}$ All cases included in the population had sonographic assessment of fetal growth within 7 days of delivery. This decision was based on prior studies that concluded that wide ultrasound-to-delivery intervals introduces bias to estimates of measurement error. ${ }^{7}$ This inclusion criteria, however, could introduce bias as both precipitous periviable deliveries as well as women with prolonged latency from ultrasound to delivery were not included in the cohort. We do not suspect that this would lead to a systematic bias, as these women were similar in baseline characteristics and indication for preterm delivery compared with subjects included in the study. Finally, we used the Hadlock-3 formula for calculation of fetal weight-which is not universally employed in all centers. This may result in differences in the accuracy of EFW. However, studies evaluating specific formulae for low BW neonates have not shown any formula to be more accurate in this particular population. ${ }^{13}$ We therefore selected the formula most commonly used in clinical practice to allow a pragmatic comparison.

In conclusion, our analysis adds additional evidence to a growing body of literature that suggests EFW is a reasonable proxy for BW among most periviable infants. It highlights, however, the limitations of ultrasound to estimate BW among SGA infants or infants less than $500 \mathrm{~g}$. Thus, clinicians should use caution when SGA is diagnosed by ultrasound in periviable fetuses, as these assessments likely underestimate the true extent of the poor fetal growth. In addition, thought should be given to using a new or different formula for infants predicted to be less than $500 \mathrm{~g}$. Given that these infants are at highest risk for poor neonatal outcomes, it is important to acknowledge the limitations of our antenatal tools to predict BW within this population.

\section{Conflict of Interest}

None.

\section{References}

1 Ecker JL, Kaimal A, Mercer BM, et al; American College of Obstetricians and Gynecologists and the Society for MaternalFetal Medicine. \#3: Periviable birth. Am J Obstet Gynecol 2015; 213(05):604-614

2 Cummings J; Committee on Fetus and Newborn. Antenatal counseling regarding resuscitation and intensive care before 25 weeks of gestation. Pediatrics 2015;136(03):588-595

3 Stoll BJ, Hansen NI, Bell EF, et al; Eunice Kennedy Shriver National Institute of Child Health and Human Development Neonatal Research Network. Neonatal outcomes of extremely preterm infants from the NICHD Neonatal Research Network. Pediatrics 2010;126(03):443-456

4 Hadlock FP, Harrist RB, Sharman RS, Deter RL, Park SK. Estimation of fetal weight with the use of head, body, and femur measurements-a prospective study. Am J Obstet Gynecol 1985;151(03): 333-337

5 Committee on Practice Bulletins-Obstetrics and the American Institute of Ultrasound in Medicine. 175: Ultrasound in pregnancy. Obstet Gynecol 2016;128(06):e241-e256

6 Dudley NJ. A systematic review of the ultrasound estimation of fetal weight. Ultrasound Obstet Gynecol 2005;25(01):80-89

7 Cohen JM, Hutcheon JA, Kramer MS, Joseph KS, Abenhaim H, Platt RW. Influence of ultrasound-to-delivery interval and maternalfetal characteristics on validity of estimated fetal weight. Ultrasound Obstet Gynecol 2010;35(04):434-441

8 Ethridge JK Jr, Louis JM, Mercer BM. Accuracy of fetal weight estimation by ultrasound in periviable deliveries. J Matern Fetal Neonatal Med 2014;27(06):557-560

9 Stefanelli S, Groom KM. The accuracy of ultrasound-estimated fetal weight in extremely preterm infants: a comparison of small for gestational age and appropriate for gestational age. Aust N Z J Obstet Gynaecol 2014;54(02):126-131

10 Alexander GR, Himes JH, Kaufman RB, Mor J, Kogan M. A United States national reference for fetal growth. Obstet Gynecol 1996;87 (02):163-168

11 Jouannic JM, Grangé G, Goffinet F, Benachi A, Carbrol D. Validity of sonographic formulas for estimating fetal weight below 1,250 g: a series of 119 cases. Fetal Diagn Ther 2001;16(04): 254-258

12 Grantz KL, Grewal J, Albert PS, et al. Dichorionic twin trajectories: the NICHD Fetal Growth Studies. Am J Obstet Gynecol 2016;215 (02):221.e1-221.e16

13 Hoopmann M, Bernau B, Hart N, Schild RL, Siemer J. Do specific weight formulas for fetuses $<$ or $=1500$ g really improve weight estimation? Ultraschall Med 2010;31(01):48-52 